\title{
Phase I trial of docetaxel, cisplatin and concurrent radical radiotherapy in locally advanced oesophageal cancer
}

\section{FL Day', T Leong', S Ngan², R Thomas ${ }^{3}$, M Jefford', JR Zalcberg', D Rischin', J McKendick ${ }^{4}$, AD Milner ${ }^{5}$, J Di lulio ${ }^{5}$, A Matera ${ }^{5}$ and M Michael $^{*, 1}$}

'Department of Medical Oncology, Peter MacCallum Cancer Centre, Locked Bag I, A'Beckett St, Melbourne, Victoria 8006, Australia; ${ }^{2}$ Department of Radiation Oncology, Peter MacCallum Cancer Centre, Locked Bag I, A'Beckett St, Melbourne, Victoria 8006, Australia; ${ }^{3}$ Division of Surgical Oncology, Peter MacCallum Cancer Centre, Locked Bag I, A'Beckett St, Melbourne, Victoria 8006, Australia; ${ }^{4}$ Department of Medical Oncology, Box Hill Hospital, Box Hill, Australia; ${ }^{5}$ Centre for Biostatistics and Clinical Trials, Peter MacCallum Cancer Centre, Locked Bag I, A'Beckett St, Melbourne, Victoria 8006, Australia

BACKGROUND: Locally advanced oesophageal cancer (LAEC) is associated with poor survival and more effective treatments are needed. The aim of this phase I trial was to assess the maximum tolerated dose (MTD) of a novel weekly docetaxel and cisplatin regimen concurrent with radical radiotherapy.

METHODS: Patients with unresectable, non-metastatic LAEC were eligible. Treatment comprised docetaxel $15-30 \mathrm{mg} \mathrm{m}^{-2}$ per week and cisplatin 15-30 $\mathrm{mg} \mathrm{m}^{-2}$ per week in six planned dose levels (DLs) in 3-6 patient cohorts with 50 Gy radiotherapy in 25 fractions. Maximum tolerated dose was based on defined dose-limiting toxicities (DLTs) during therapy and 2 weeks post therapy. RESULTS: A total of 24 patients were enrolled. There were two DLTs: grade 3 fever in DLI (docetaxel $15 \mathrm{mg} \mathrm{m}^{-2}$, cisplatin $\left.15 \mathrm{mg} \mathrm{m}^{-2}\right)$ and grade 3 nausea in DL2 $\left(20 \mathrm{mg} \mathrm{m}^{-2}, 15 \mathrm{mg} \mathrm{m}^{-2}\right)$. These DLs were each expanded to six patients without further DLTs. The most common acute toxicity was grade 3 radiation oesophagitis (37.5\%). There were no grade 4 toxicities, and haematologic toxicity was minimal. Cisplatin and docetaxel dose intensity was 100\% at the highest dose level (DL6). A MTD was not reached in this trial. Tumour overall response rate was 50\% (33\% complete, $17 \%$ partial).

CONCLUSION: Cisplatin and docetaxel each $30 \mathrm{mg} \mathrm{m}^{-2}$ per week concurrent with $50 \mathrm{~Gy}$ radiotherapy is recommended for use in phase II clinical trials in oesophageal cancer.

British Journal of Cancer (20II) I 04, 265-27I. doi: I0.1038/sj.bjc.660605 I www.bjcancer.com

Published online 14 December 2010

(C) 20II Cancer Research UK

Keywords: oesophageal cancer; chemoradiotherapy; docetaxel; cisplatin

Oesophageal cancer is an important global health problem, ranking 7th among male cancer deaths in the United States and increasing in incidence (Jemal et al, 2009). The 5-year survival rates are poor, approximately $17 \%$ in the period 1996-2004 (Jemal et al, 2009). Surgical resection, with neoadjuvant chemotherapy or chemoradiotherapy (Gebski et al, 2007), confers the greatest likelihood of long-term survival, but only $30-40 \%$ of patients with newly diagnosed oesophageal cancer have resectable disease. For patients with unresectable, locally advanced oesophageal cancer (LAEC) (due to advanced $\mathrm{T}$ or $\mathrm{N}$ stage, or patient medically unsuitable for surgery), chemoradiotherapy is the mainstay of treatment.

The most commonly used chemotherapy for radiosensitisation in LAEC, and in palliation, are the platinums and 5-fluorouracil. The RTOG 85-01 trial randomised patients with LAEC to receive cisplatin and infusional 5-fluorouracil concurrently with $50 \mathrm{~Gy}$ or 64 Gy radiotherapy alone. This study showed a significantly improved 5-year survival of $27 \%(v s 0 \%)$ for patients receiving

\footnotetext{
* Correspondence: Associate Professor M Michael;

E-mail: Michael.Michael@petermac.org

Received 25 June 2010; revised 9 November 2010; accepted 16 November 2010; published online 14 December 2010
}

chemoradiation (Herskovic et al, 1992; Al-Sarraf et al, 1997). Nevertheless, outcomes were still poor in the combined modality arm, with $25 \%$ patients having persistent disease, and 2-year rates of local recurrence and distant disease of 45 and $21 \%$, respectively. New active therapies and treatment approaches in oesophageal cancer are warranted.

Docetaxel is a semi-synthetic taxane with promising single-agent activity in advanced oesophageal cancer (Einzig et al, 1996), and greater activity in in vitro oesophageal cancer studies than paclitaxel (Kawamura et al, 1997). Docetaxel is a potent radiosensitiser through promotion of microtubule stability, causing arrest in G2 and M phases of the cell cycle and, hence, sensitivity to radiation injury (Mason et al, 1997). A phase I trial of docetaxel concurrent with $60 \mathrm{~Gy}$ thoracic radiotherapy in patients with oesophageal and non-small-cell lung cancer (NSCLC) (Mauer et al, 1998 ) found the maximum tolerated dose (MTD) of docetaxel to be $20 \mathrm{mg} \mathrm{m}^{-2}$ per week. The dose limiting toxicity (DLT) for the weekly regimen in this study was grades 3 and 4 oesophagitis. Local and systemic disease control could potentially be enhanced by the addition of cisplatin, given its known activity in oesophageal cancer and non-overlapping toxicity profile.

In this phase I trial, we assessed a novel regimen of weekly docetaxel and cisplatin concurrent with radiotherapy (50 Gy) for patients with LAEC. The primary objective was to define the MTD 
of this chemotherapy combination with concurrent radical radiotherapy, with a view to establishing a recommended dose level for future multimodality clinical trials. The secondary objectives were to define the safety (acute and long term) of this regimen when combined with radical radiotherapy, as well as determine the response rate, overall survival (OS) and progression-free survival (PFS) of the patients treated with this protocol.

\section{PATIENTS AND METHODS}

\section{Patients}

Patients were eligible if they met the following criteria: (1) histologically proven squamous cell carcinoma or adenocarcinoma of the oesophagus; (2) considered unsuitable for surgical resection (due to advanced $\mathrm{T}$ or $\mathrm{N}$ stage, or patient medically unsuitable for surgery) based on multidisciplinary opinion; (3) no previous therapy for oesophageal cancer; (4) ECOG performance status (PS) of zero or one; (5) life expectancy $>3$ months; (6) adequate organ function - (i) hepatic: serum bilirubin $\leqslant 1.0 \times$ upper limit normal $(\mathrm{ULN}), \mathrm{AST}$ and/or ALT $\leqslant 2.0 \times \mathrm{ULN}, \mathrm{ALP} \leqslant 2.5 \times \mathrm{ULN}$, (ii) bone marrow: haemoglobin $\geqslant 100 \mathrm{gl}^{-1}$, neutrophil count $\geqslant 1.5 \times 10^{9}$ per $\mathrm{l}$, platelet count $\geqslant 100 \times 10^{9}$ per 1 , (iii) renal: creatinine clearance $\geqslant 55 \mathrm{ml} \mathrm{min}^{-1}$ (using radioisotope renal scan or derived from serum creatinine using the Cockroft-Gault formula); (7) agreed compliance to adequate contraception; (8) written informed consent.

The following patients were ineligible: (1) resectable oesophageal carcinoma; (2) carcinoma of the cervical oesophagus; (3) tumour predominantly in the stomach; (4) metastatic oesophageal carcinoma; (5) medical comorbidities which would compromise the delivery of therapy or which may be exacerbated by the planned treatment; (6) receiving treatment with another investigational agent; (7) pregnant or lactating females.

Institutional ethics committee approval was obtained from each participating site.

\section{Treatment plan}

Radiation therapy External beam radiotherapy was given to a total dose of $50 \mathrm{~Gy}$ in 25 fractions, five fractions per week for 5 weeks. Treatment was delivered using conformal techniques in accordance with ICRU 50/62 recommendations. In most cases, a two-phase technique was employed, comprising AP-PA fields to a dose of $36 \mathrm{~Gy}$ in 18 fractions, followed by lateral fields to a dose of $14 \mathrm{~Gy}$ in 7 fractions. The gross tumour volume (GTV) comprised the primary tumour and involved lymph nodes as defined by imaging, endoscopy and biopsy. The clinical target volume (CTV) was generated by applying standard margins $(5 \mathrm{~mm}$ radially and $4 \mathrm{~cm}$ longitudinally) to the GTV, and the planning target volume (PTV) was then generated by applying a volumetric $10 \mathrm{~mm}$ margin.

Concurrent chemotherapy During radiotherapy, all patients received intravenous docetaxel and cisplatin administered weekly (days 1, 8, 15, 22 and 29). Chemotherapy doses were escalated through six planned dose levels (DLs) as shown in Table 1. Chemotherapy was given within $4 \mathrm{~h}$ before the delivery of radiation that day. Steroid and anti-emetic pre-medication was administered to all patients.

Definition of dose-limiting toxicities (DLTs) The following toxicities (as per NCI-CTC version 2.0) occurring during or up to 2 weeks after chemoradiotherapy were defined prospectively as DLTs: (1) grade 4 neutropaenia (neutrophil count $<0.5 \times 10^{9}$ per 1 ) of any duration; (2) grade 3 neutropaenia of any duration during chemoradiotherapy; (3) grade 4 thrombocytopaenia (platelet count $<10 \times 10^{9}$ per l) of any duration; (4) grade 3 thrombocytopaenia $\left(10-49 \times 10^{9}\right.$ per 1$)$ with bleeding; (5) febrile neutropaenia;
Table I Chemotherapy dose levels

\begin{tabular}{lccc}
\hline $\begin{array}{l}\text { Dose level } \\
\text { (DL) }\end{array}$ & $\begin{array}{c}\text { Docetaxel weekly } \\
\left(\mathbf{m g ~ m}^{\mathbf{- 2}} \mathbf{)}\right.\end{array}$ & $\begin{array}{c}\text { Cisplatin weekly } \\
\mathbf{( m g ~ m}^{-\mathbf{2}} \mathbf{)}\end{array}$ & Participants \\
\hline $0^{\mathrm{a}}$ & 10 & 10 & 6 \\
1 & 15 & 15 & 6 \\
2 & 20 & 15 & 3 \\
3 & 20 & 20 & 3 \\
4 & 25 & 20 & 3 \\
5 & 30 & 20 & 3 \\
\hline
\end{tabular}

ancluded in protocol for patients who require dose reduction below DLI.

(6) grade 4 radiation oesophagitis; (7) any non-oesophagitis grade 3 or 4 radiotherapy toxicity; (8) any clinically significant treatment-related grade 3 or 4 toxicity outside the radiotherapy field, with the exception of alopecia; (9) interruption of radiotherapy for $>1$ week; (10) omission of chemotherapy for $\geqslant 1$ week; (11) toxicity requiring $\geqslant 2$ chemotherapy dose reductions.

Dose escalation schema Three patients were entered into each DL. If no DLTs were observed, the next DL was opened. If a DLT was observed in one of three patients, then three additional patients (a total of six) were accrued at this level. If DLTs were observed in one of six patients, then escalation to the next level took place. If DLTs were observed in $\geqslant 2$ of 3 or $\geqslant 2$ of 6 patients, then no further dose escalation took place. Maximum tolerated dose was predefined as the second highest dose level reached.

Dose escalation to the next DL, or expansion of the current DL, only occurred when all three patients at the current DL had completed chemoradiotherapy and 2 weeks post-therapy. There was no intra-patient dose escalation.

Dose modifications during chemoradiotherapy Radiotherapy, together with chemotherapy, was suspended if the patient experienced grade 4 oesophagitis, or grade 3 or 4 other radiation-associated toxicity. Treatment could recommence once reactions had improved to grade 1, unless treatment was interrupted for 2 or more weeks, in which case all therapy was to be ceased.

Prospectively defined chemotherapy dose modifications (dose omission, delay or reduction) were based on the worst grades of haematological and non-haematological toxicity during chemoradiotherapy. All dose reductions were permanent. Treatment was ceased if grade 3 or 4 neurological toxicity, other grade 4 nonhaematological toxicity, more than one dose reduction required in DL1, or more than two dose reductions required in higher DLs. Radiotherapy continued despite chemotherapy modifications.

\section{Monitoring procedures and tests}

At baseline (within 1 week of study entry), patients underwent physical examination, assessment of PS, and blood taken for full blood examination (FBE), biochemistry (including serum urea, creatinine, electrolytes, calcium, liver function tests (bilirubin, AST, ALT, ALP, GGT)) and determination of creatinine clearance. Within 4 weeks before study entry, patients underwent endoscopy, computed tomography (CT) scan of the chest/abdomen and wholebody bone scan. Optional baseline tests were endoscopic ultrasound, bronchoscopy and FDG-PET scan (subject to availability).

During treatment, patients were reviewed weekly, had their weight measured, PS assessed, physical examination and recording of acute toxicities. Blood was taken for FBE twice per week, and biochemistry and creatinine clearance measured weekly.

Following completion of chemoradiotherapy, patients were assessed clinically (physical examination, body weight and PS) at weeks 1, 2, 4 and 6 . Full blood examination and biochemistry were 
measured at weeks 1 and 2. Patients underwent repeat endoscopy and CT scan of the chest/abdomen at 6 weeks, and FDG-PET at $8-10$ weeks (subject to availability).

Follow-up evaluation then occurred every 3 months until patient death or loss to follow-up. This comprised clinical examination, assessment of late radiation toxicity and repeat CT scan of chest/ abdomen.

\section{Statistical methods}

Acute toxicity was graded and reported according to the NCI-CTC version 2.0 (30 April 1999). Late radiation toxicities were assessed using the RTOG/EORTC criteria. RECIST version 1.0 was used for assessment of radiological response.

Progression-free survival was measured from treatment start date until disease progression or death from any cause. Overall survival was measured from treatment start date until death from any cause. Progression-free survival and OS were determined using the Kaplan-Meier method, with times censored at the close-out date for patients still being followed without evidence of disease/ death, or the date of last contact for those patients lost to follow-up. Using Logit transformation 95\% confidence intervals were calculated. All other data were summarised using descriptive statistics, including counts and percentages for categorical data and the median and range for continuous data. Analyses were conducted using StatXact (Version 5.0.3, Cytel Software Corporation, Cambridge, MA, USA; 2001) and S-Plus 2000 (MathSoft Inc., Seattle, WA, USA; 1999).

\section{RESULTS}

\section{Patients}

A total of 24 patients were recruited from three centres in Melbourne, Australia, between May 2001 and January 2007; Peter MacCallum Cancer Centre, Box Hill Hospital and Monash Medical Centre. The majority of patients were ineligible for surgical resection on the basis of nodal involvement. Of the optional staging tests, 11 patients received a baseline FDG-PET scan, 4 patients underwent endoscopic ultrasound and 3 patients bronchoscopy. Patient demographics are summarised in Table 2. Median patient age was 58 years and males predominated (92\%). One patient had undergone previous surgery for oesophageal carcinoma.

\section{Treatment delivery}

Radiotherapy A total of 23 patients (96\%) completed all planned radiotherapy without any treatment interruptions. One patient did not complete the final two fractions of radiotherapy because of grade 3 radiation oesophagitis and fever. Three patients received $50.4 \mathrm{~Gy}$ in 28 fractions, rather than the protocol specified $50 \mathrm{~Gy}$ in 25 fractions, because of investigator prescription error.

Chemotherapy The 24 accrued patients were treated across six DLs as shown in Table 1. DLs one and two were each expanded to six patients because of the occurrence of a DLT in one of the three initial patients.

Only one patient did not receive the protocol-defined chemotherapy doses. This patient was in DL3 and had cisplatin omitted on days 15 and 22 because of creatinine clearance $<55 \mathrm{ml} \mathrm{min}^{-1}$ on those dates. No docetaxel doses were omitted. One patient (DL1) had a 2-day delay in chemotherapy administration because of hospitalisation with a DLT. The relative dose intensity (ratio of actual dose to planned dose) in the highest dose level (DL6) was $100 \%$ for both cisplatin and docetaxel.
Table 2 Patient characteristics

\begin{tabular}{|c|c|c|}
\hline Characteristics & Number $(n=24)$ & $\%$ \\
\hline \multicolumn{3}{|l|}{ Sex } \\
\hline Male: female & $22: 2$ & $92: 8$ \\
\hline \multicolumn{3}{|l|}{ Age (years) } \\
\hline Median & 58 & \\
\hline Range & $36-83$ & \\
\hline \multicolumn{3}{|c|}{ ECOG performance status } \\
\hline 0 & 7 & 29.2 \\
\hline । & 17 & 70.8 \\
\hline \multicolumn{3}{|l|}{ Histology } \\
\hline Squamous & ।1 & 45.8 \\
\hline Adenocarcinoma & 13 & 54.2 \\
\hline \multicolumn{3}{|l|}{ Tumour location } \\
\hline Upper third & 3 & 12.5 \\
\hline Middle third & 5 & 20.8 \\
\hline Lower third & 16 & 66.7 \\
\hline \multicolumn{3}{|l|}{$T$ stage } \\
\hline 1 & 3 & 12.5 \\
\hline 2 & 3 & 12.5 \\
\hline 3 & 16 & 66.7 \\
\hline 4 & 2 & 8.3 \\
\hline \multicolumn{3}{|l|}{$N$ stage } \\
\hline 0 & 6 & 25.0 \\
\hline 1 & 18 & 75.0 \\
\hline \multicolumn{3}{|l|}{ Stage grouping } \\
\hline 1 & 2 & 8.3 \\
\hline \|la & 4 & 16.7 \\
\hline$\| \mathrm{lb}$ & 2 & 8.3 \\
\hline III & 16 & 66.7 \\
\hline \multicolumn{3}{|l|}{ Previous surgery } \\
\hline Yes & 1 & 4.2 \\
\hline No & 23 & 95.8 \\
\hline \multicolumn{3}{|c|}{ Weight loss over previous 3 months } \\
\hline None & 9 & 37.5 \\
\hline$\leqslant 10 \%$ & II & 45.8 \\
\hline$>10 \%$ & 4 & 16.7 \\
\hline
\end{tabular}

Abbreviation: ECOG = eastern cooperative oncology group.

Dose-limiting toxicities Two DLTs were observed. One patient in DL1 experienced grade 3 fever, without neutropaenia. This patient was concurrently suffering from grade 3 oesophagitis, and was hospitalised for intravenous antibiotics and analgesia. This event was the cause of the only delay in chemotherapy administration and the only omission of radiotherapy. Dose level 1 was expanded to six patients as a result, but no further DLTs were observed.

The second DLT was in DL2, and consisted of grade 3 nausea despite anti-emetic treatment. Modification of chemoradiotherapy was not indicated, but DL2 also expanded to six patients. No further DLTs were observed, and hence a MTD was not defined.

\section{Toxicity}

There were two grade 3 haematologic toxicity events; both grade 3 leucopaenia. The first event was in DL2 and occurred at day 40 (after completion of chemoradiotherapy). The second was in DL6 and occurred at day 30, one day after final chemotherapy dosing.

Non-haematologic toxicity is shown in Table 3 . In all, $37.5 \%$ of patients experienced grade 3 dysphagia attributed to radiation 
Table 3 Non-haematologic toxicities observed during chemoradiotherapy and within 2 weeks after completion, and considered possibly, probably or definitely related to treatment ( $\mathrm{NCl}-\mathrm{CTC}$ Version 2, 30 April 1999)

Dose level

\begin{tabular}{|c|c|c|c|c|c|c|c|c|}
\hline Toxicity & Worst grade & I $(n=6)$ & $2(n=6)$ & $3(n=3)$ & $4(n=3)$ & $5(n=3)$ & $6(n=3)$ & Total (\%) \\
\hline \multicolumn{9}{|c|}{ Within radiotherapy field } \\
\hline Dysphagia & 3 & 2 & 3 & 1 & 0 & 2 & 1 & $9(37.5)$ \\
\hline \multicolumn{9}{|c|}{ Outside radiotherapy field } \\
\hline Anorexia & 3 & 0 & I & 0 & 0 & 0 & 0 & I (4.2) \\
\hline Nausea & 3 & 0 & 2 & 0 & 0 & 0 & । & $3(12.5)$ \\
\hline Constipation & 3 & 1 & 0 & 1 & 0 & 0 & 1 & $3(12.5)$ \\
\hline Fatigue & 3 & I & I & 2 & 0 & 0 & 0 & $4(16.7)$ \\
\hline Fever & 3 & $1^{a}$ & 0 & 0 & 0 & 0 & 0 & I (4.2) \\
\hline \multicolumn{9}{|l|}{ Biochemical } \\
\hline Hyperglycaemia & 3 & 0 & I & 0 & 0 & 0 & 0 & I (4.2) \\
\hline Hyponatraemia & 3 & 0 & 1 & 0 & 0 & I & 0 & $2(8.3)$ \\
\hline Elevated GGT & 4 & $2^{b}$ & 0 & 0 & 0 & I & 0 & $3(12.5)$ \\
\hline
\end{tabular}

aithout neutropenia. ${ }^{\mathrm{b}}$ One patient in DLI experienced grade 4 elevation of GGT, attributed to penicillin antibiotics, and reversed on their cessation.

Table 4 Late radiotherapy toxicities (EORTC/RTOG criteria)

Dose level

\begin{tabular}{|c|c|c|c|c|c|c|c|c|}
\hline Toxicity & Worst grade & I $(n=6)$ & $2(n=6)$ & $3(n=3)$ & $4(n=3)$ & $5(n=3)$ & $6(n=3)$ & Total (\%) \\
\hline Skin & 2 & 2 & 0 & 0 & 0 & I & 0 & $3(12.5)$ \\
\hline
\end{tabular}

Table 5 Radiologic response at completion of chemoradiotherapy (RECIST version I.0)

\section{Dose levels}

\begin{tabular}{|c|c|c|c|c|c|c|c|}
\hline Response parameters & I $(n=6)$ & $2(n=6)$ & $3(n=3)$ & $4(n=3)$ & $5(n=3)$ & $6(n=3)$ & $\begin{array}{c}\text { No. }(\%) \\
(\text { Total } n=24)\end{array}$ \\
\hline \multicolumn{8}{|l|}{ Overall best response } \\
\hline Complete response & 4 & 1 & 0 & 2 & 1 & 0 & $8(33)$ \\
\hline Partial response & 1 & 3 & 0 & 0 & 0 & 0 & $4(17)$ \\
\hline Stable disease & 0 & 0 & 3 & 0 & 1 & 1 & $5(21)$ \\
\hline Progressive disease & 1 & 2 & 0 & 0 & 1 & 1 & $5(21)$ \\
\hline Not evaluable ${ }^{a}$ & 0 & 0 & 0 & 1 & 0 & 1 & $2(8)$ \\
\hline \multicolumn{8}{|c|}{ Best response in radiotherapy field } \\
\hline Complete response & 4 & 2 & 0 & 2 & I & 0 & $9(37)$ \\
\hline Partial response & 1 & 3 & 0 & 0 & 0 & 0 & $4(17)$ \\
\hline Stable disease & 0 & 0 & 3 & 0 & I & 1 & $5(21)$ \\
\hline Progressive disease & 1 & I & 0 & 0 & 0 & 1 & $3(13)$ \\
\hline Not evaluable & 0 & 0 & 0 & I & I & 1 & $3(13)$ \\
\hline
\end{tabular}

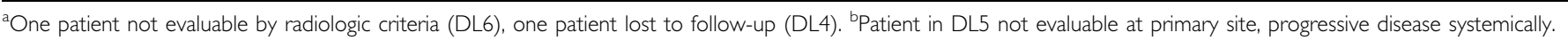

oesophagitis. There were no episodes of grade 4 oesophagitis and no acute pulmonary toxicity. Outside the radiotherapy field the most common adverse event was fatigue.

Late radiation toxicities are shown in Table 4

\section{Response}

In all, 21 of 24 patients were evaluable for radiologic response within the radiation field (Table 5). The within-field response rate was $54 \%$ (37\% complete and $17 \%$ partial), and $21 \%$ of patients had stable disease. Three patients (13\%) had progressive disease within the radiotherapy field, all concurrent with distant progression.
The overall response rate to treatment, incorporating systemic disease progression, was $50 \%$ (Table 5 ).

A total of 11 patients underwent FDG-PET at baseline, and 10 of these also progress scans following treatment. Four patients experienced a complete metabolic response (40\%), five a partial metabolic response $(50 \%)$ and one patient progressive disease $(10 \%)$.

\section{Further treatment post-chemoradiotherapy}

Six patients (25\%) later underwent oesophageal surgery with curative intent, range 56-283 days after completing chemoradiotherapy. Surgery performed was Ivor-Lewis oesophagectomy 


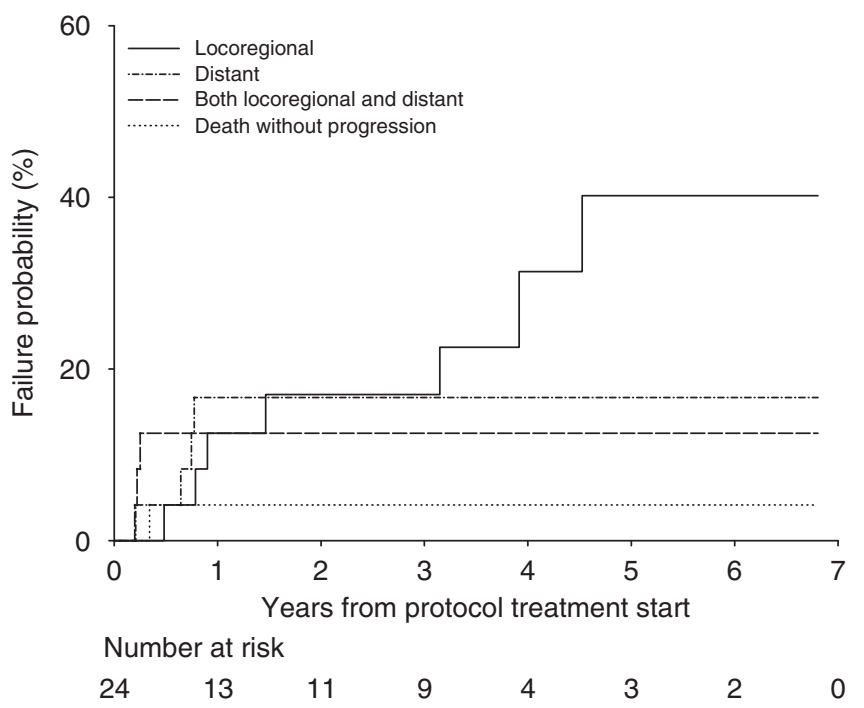

Figure I Site of first failure in patients with recurrent disease $(n=14)$.

in four patients, three-stage oesophagectomy in one patient and total oesophagectomy in one patient.

\section{Sites of relapse}

Patients were followed to a close-out date of 16 June 2010. One patient was lost to follow-up after completing protocol treatment and hence was not evaluable for treatment response, but has been included in survival analyses as the date of death is known. Median patient follow-up was 5.2 years. Disease relapse was reported in 14 patients. The location of first disease progression is shown in Figure 1. In all, $42 \%$ of enrolled patients (10 of 24 ) experienced initial local disease relapse (primary site and/or regional nodes) and $29 \%$ (7 of 24 ) distant metastases.

\section{Survival parameters}

Kaplan-Meier estimates of PFS are shown in Figure 2. Median PFS was 1.46 years (95\% CI $0.75-6.81$ years). Progression-free survival at 2 years was $49.7 \%$ (95\% CI $30.6-68.8 \%)$, and $26.5 \%$ at 5 years $(95 \%$ CI 10.1-53.5\%). Median OS was 4.02 years (95\% CI 1.08-6.81 years, Figure 3). Overall survival at 2 years and 5 years was $57.8 \%(95 \%$ CI $37.6-75.6 \%)$ and $30.5 \%$ (95\% CI $13.1-56.2 \%)$, respectively.

\section{DISCUSSION}

The semi-synthetic taxane docetaxel has shown activity in oesophageal cancer in numerous clinical trials. Docetaxel has been used in advanced oesophageal cancer as a single agent (Einzig et al, 1996; Heath et al, 2002), and in combination with cisplatin (Laack et al, 2005), 5-fluorouracil (Chun et al, 2001), capecitabine (Lorenzen et al, 2005) and irinotecan (Lordick et al, 2003). Known to be a potent radiosensitiser, docetaxel has also been used in chemoradiotherapy for oesophageal cancer (Mauer et al, 2000; Pasini et al, 2005; Font et al, 2007; Ruhstaller et al, 2009). The initial phase I trial of single agent docetaxel with thoracic radiotherapy (Mauer et al, 1998) included nine patients with oesophageal cancer (total $n=29$, remainder NSCLC) and tested docetaxel in escalating total doses of 40,60 or $75 \mathrm{mg} \mathrm{m}^{-2}$ (given in one, two or three divided doses) per 21-day cycle and concurrent with median $60 \mathrm{~Gy}$ radiotherapy. Dose-limiting toxicities in this trial were radiation oesophagitis and febrile neutropaenia; the docetaxel MTD was $20 \mathrm{mg} \mathrm{m}^{-2}$ per week. This docetaxel dose and

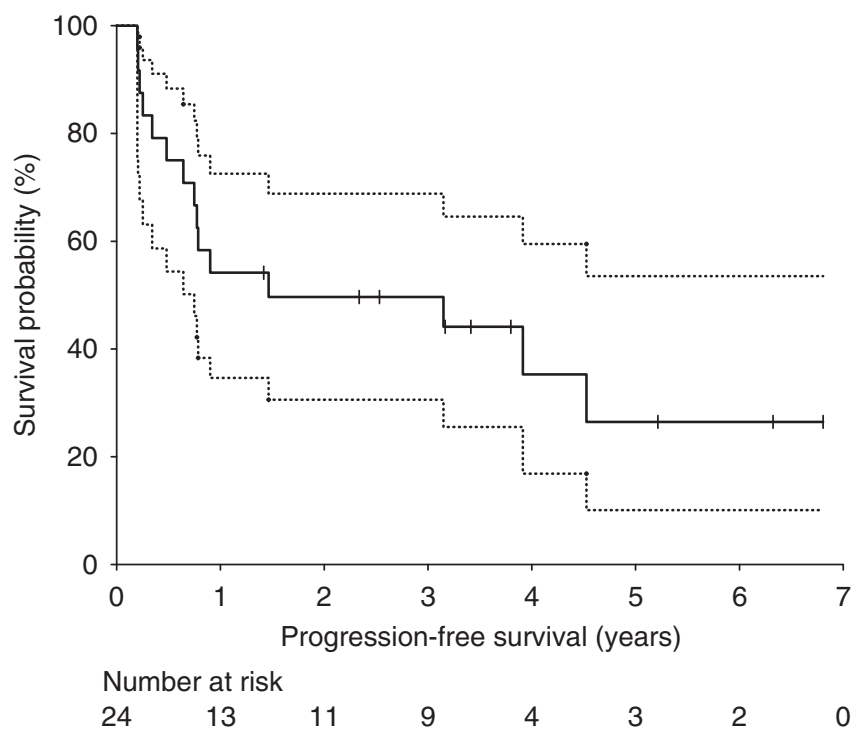

Figure 2 Kaplan-Meier curves for progression-free survival for all patients. Dotted lines represent the $95 \% \mathrm{Cl}$. Vertical lines represent patients censored at the close-out date.

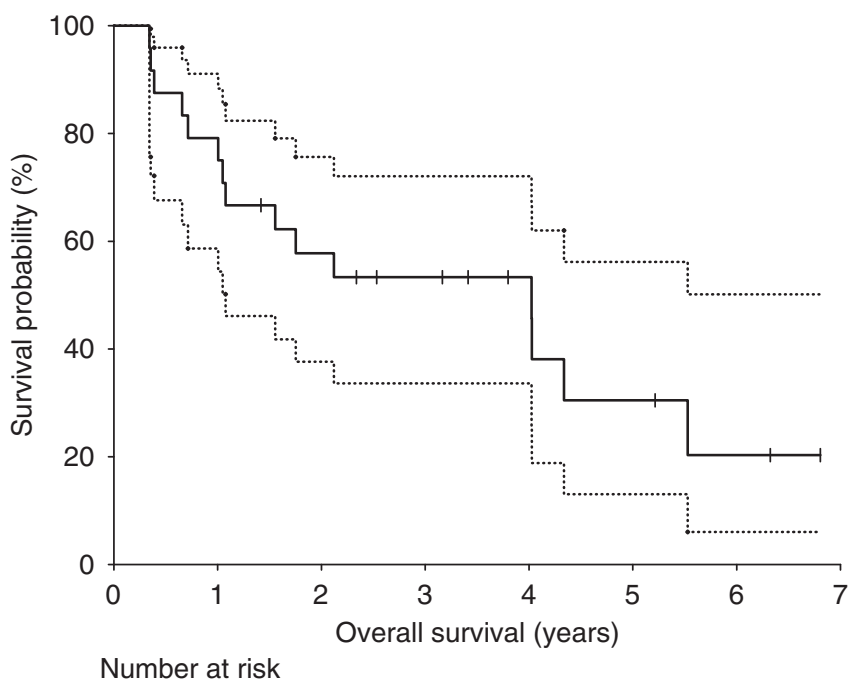

$\begin{array}{llllllll}24 & 19 & 13 & 10 & 7 & 4 & 2 & 0\end{array}$

Figure 3 Kaplan-Meier curves for overall survival for all patients. Dotted lines represent the $95 \% \mathrm{Cl}$. Vertical lines represent patients censored at the close-out date.

schedule was then tested in a phase II trial (Font et al, 2007) in 34 patients with oesophageal cancer unsuitable for surgical resection based on T4 status or co-morbidities, and given concurrently with $66 \mathrm{~Gy}$ radiotherapy. Radiologic response rates were $26 \%$ complete and $24 \%$ partial. Median OS was 6 months, with $35 \%$ and $12 \%$ patients alive at 1 and 3 years, respectively. The rate of grade 3 and 4 radiation oesophagitis was $17 \%$. These phase I and II trials (Mauer et al, 1998; Font et al, 2007) of single agent docetaxel with 60-66 Gy radiotherapy each had two deaths from radiation pneumonitis.

Chemoradiotherapy using both docetaxel and cisplatin has been the subject of phase I trials in NSCLC. Although the DLT for each published study was radiation oesophagitis, variable MTDs are reported; docetaxel $20 \mathrm{mg} \mathrm{m}^{-2}$ per week with cisplatin fixed at $20 \mathrm{mg} \mathrm{m}^{-2}$ per week and $63 \mathrm{~Gy}$ radiotherapy (Wu et al, 2002), docetaxel $25 \mathrm{mg} \mathrm{m}^{-2}$ per week with cisplatin fixed at $25 \mathrm{mg} \mathrm{m}^{-2}$ per 
week and 60 Gy (Mudad et al, 2003), and docetaxel and cisplatin both at $40 \mathrm{mg} \mathrm{m}^{-2}$ per week with $60 \mathrm{~Gy}$ (Kiura et al, 2003). The latter study was expanded to enroll 42 patients in the phase II setting, and found an excellent response rate of $79 \%$ in stage III NSCLC, but high rates of grade 3 and 4 myelosuppression (Kiura et al, 2003). A subsequent study by the same authors used this regimen (cisplatin $40 \mathrm{mg} \mathrm{m}^{-2}$ per week, docetaxel $40 \mathrm{mg} \mathrm{m}^{-2}$ per week, $60 \mathrm{~Gy}$ radiotherapy) as induction treatment before resection in locally advanced NSCLC, and found that 11 of 22 patients $(50 \%)$ were unable to complete chemotherapy without dose modification because of toxicity (Katayama et al, 2004).

Pasini et al (2005) conducted a phase I study in localised oesophageal cancer using induction weekly docetaxel, cisplatin and infusional 5-fluorouracil, followed by escalating doses of the same three agents concurrent with up to $50 \mathrm{~Gy}$ radiotherapy, then surgery. Maximum tolerated dose was not reached at the highest planned dose level; docetaxel $35 \mathrm{mg} \mathrm{m}^{-2}$ per week, cisplatin $25 \mathrm{mg} \mathrm{m}^{-2}$ per week and infusional 5 -FU $150 \mathrm{mg} \mathrm{m}^{-2}$ per day with $50 \mathrm{~Gy}$ radiotherapy. These doses are the subject of an ongoing phase II trial by the same group. A recently published Swiss multicentre phase II trial used induction docetaxel and cisplatin (75 $\mathrm{mg} \mathrm{m}^{-2}$ each, two 21-day cycles), followed by weekly concurrent treatment $\left(20 \mathrm{mg} \mathrm{m}^{-2}\right.$ and $25 \mathrm{mg} \mathrm{m}^{-2}$, respectively) with $45 \mathrm{~Gy}$ radiotherapy, then surgery, in locally advanced but resectable oesophageal cancer (Ruhstaller et al, 2009). Concurrent chemoradiotherapy resulted in grade 3 and 4 dysphagia in only $8 \%$ of patients and grade 3 and 4 anaemia, thrombocytopenia and neutropenia each in less than $3 \%$. In all, $86 \%$ of patients proceeded to surgical resection and $\mathrm{R} 0$ resection was achieved in $79 \%$ enrolled patients.

The phase I trial reported here used 50 Gy radiotherapy with the goal of optimising systemic therapy delivery and radiosensitisation without dose-limiting radiotherapy toxicity. Cisplatin was added to docetaxel because of its established role as a radiosensitiser in oesophageal cancer and minimal additional myelotoxicity. In all, 9 of 24 patients $(37.5 \%)$ experienced grade 3 radiation oesophagitis, but there were no episodes of grade 4 oesophagitis. Other than the two reported DLTs, toxicities were low grade, not probably/ definitely related to treatment, or deemed not clinically significant. There were no radiation pneumonitis events, and haematologic toxicity from the weekly chemotherapy was negligible, consistent with the known lower myelotoxicity of this docetaxel schedule (Hainsworth et al, 1998). The maximum severity late toxicity was grade 2 and skin related. Only one patient did not receive $100 \%$ of prescribed chemotherapy and radiotherapy doses because of toxicity, and the maximum protocol cisplatin and docetaxel doses (DL6) were given without achieving a MTD as predefined in the trial protocol, as also observed by Pasini et al (2005) with a similar backbone and doses, but with the addition of infusional 5-FU.
Like Pasini et al (2005), we elected not to increase chemotherapy doses above the pre-planned levels, despite not achieving a MTD, because of expected prohibitive toxicity with further escalation. The highest DL in this trial exceeds the MTDs established in concurrent chemoradiotherapy for NSCLC (Wu et al, 2002; Mudad et al, 2003), other than the study by Kiura et al (2003), which has subsequently shown significant toxicity, and the cisplatin and docetaxel doses are also greater than those used in the phase II oesophageal cancer trial by Ruhstaller et al (2009).

Although not powered to demonstrate improvements in cancer outcomes, the radiologic response rate of $54 \%$ in the radiotherapy field, and $50 \%$ overall, in this trial compares favourably with phase II chemoradiotherapy trials (Font et al, 2007; 50\%), including those also using induction chemotherapy before docetaxel-based chemoradiotherapy (Mauer et al, 2000; 58\%). The median PFS and OS of 1.5 and 4.0 years presented here are surprisingly separate, given the median survival time of 8 months with recurrent oesophageal cancer (Meguid et al, 2009); this apparent discrepancy is because of the effect of small patient numbers on survival curves. Nonetheless, these survival times are both considerably longer than those of the landmark RTOG 85-01 trial cisplatin/ 5-fluorouracil/radiotherapy arm (1 year and 14.1 months, respectively). Participants in this study were median age 6 years younger than those in RTOG 85-01, better PS and different in tumour histology $(54 \%$ vs $12 \%$ adenocarcinoma) and site (greater proportion lower oesophagus), but significantly higher $\mathrm{T}$ and $\mathrm{N}$ stage. The subsequent use of curative-intent surgery in six patients $(25 \%)$ in the current trial may have contributed to the improved survival outcomes. The predominance of locoregional recurrence over distant disease progression in this trial is similar to that seen in the randomized chemoradiotherapy arm of RTOG 85-01, postulated by those authors as reflecting an effect of chemotherapy on micrometastatic disease (Al-Sarraf et al, 1997).

In conclusion, the phase I trial presented here has shown that weekly administration of cisplatin and docetaxel to each $30 \mathrm{mg} \mathrm{m}^{-2}$ per week concurrent with $50 \mathrm{~Gy}$ radiotherapy is tolerable and deliverable in locally advanced oesophageal cancer. These results have led to the incorporation of this chemoradiotherapy backbone into a current phase II study of the Australasian Gastro-Intestinal Trials Group (AGITG) in resectable oesophageal cancer with a randomisation to the addition of cetuximab.

\section{ACKNOWLEDGEMENTS}

We would like to thank Sanofi-Aventis Australia for the provision of docetaxel chemotherapy.

\section{REFERENCES}

Al-Sarraf M, Martz K, Herskovic A, Leichman L, Brindle JS, Vaitkevicius VK, Cooper J, Byhardt R, Davis L, Emami B (1997) Progress report of combined chemoradiotherapy versus radiotherapy alone in patients with esophageal cancer: An intergroup study. J Clin Oncol 15: 277-284

Chun HG, Puccio CA, Mittelman A (2001) Weekly docetaxel and continuous infusion (CI) 5-fluorouracil (5-FU) in elderly patients (Pts) with cancer of the stomach and distal esophagus. Proc Am Soc Clin Oncol 20: 2001 (abstract 645)

Einzig AI, Neuberg D, Remick SC, Karp DD, O'Dwyer PJ, Stewart JA, Benson III AB (1996) Phase II trial of docetaxel (Taxotere) in patients with adenocarcinoma of the upper gastrointestinal tract previously untreated with cytotoxic chemotherapy: the Eastern Cooperative Oncology Group (ECOG) results of protocol E1293. Med Oncol 13: 87-93

Font A, Arellano A, Fernández-Llamazares J, Casas D, Boix J, Cardenal J, Margeli M, Manzano JL, Abad A, Rosell R (2007) Weekly docetaxel with

concomitant radiotherapy in patients with inoperable oesophageal cancer. Clin Trans Oncol 9: 177-182

Gebski V, Burmeister B, Smithers BM, Foo K, Zalcberg J, Simes J (2007) Survival benefits from neoadjuvant chemoradiotherapy or chemotherapy in oesophageal carcinoma: a meta-analysis. Lancet Oncol 8: 226-234

Hainsworth JD, Burris HA, Erland JB, Thomas M, Greca FA (1998) Phase I trial of docetaxel administered by weekly infusion in patients with advanced refractory cancer. J Clin Oncol 16: 2164-2168

Heath EI, Urba A, Marshall J, Piantadosi S, Forastiere AA (2002) Phase II trial of docetaxel chemotherapy in patients with incurable adenocarcinoma of the esophagus. Invest New Drugs 20: 95-99

Herskovic A, Martz K, Al-Sarraf M, Leichman L, Brindle J, Vaitkevicius V, Cooper J, Byhardt R, Davis L, Emami B (1992) Combined chemotherapy and radiotherapy compared with radiotherapy alone in patients with cancer of the esophagus. N Engl J Med 326: $1593-1598$ 
Jemal A, Siegel R, Ward E, Hao Y, Xu J, Thun MJ (2009) Cancer statistics, 2009. CA Cancer J Clin 59: 225-249

Katayama $\mathrm{H}$, Ueoka $\mathrm{H}$, Kiura $\mathrm{K}$, Tabata $\mathrm{M}$, Kozuki $\mathrm{T}$, Tanimoto $\mathrm{M}$, Fujiwara T, Tanaka N, Date H, Aoe M, Shimizu N, Takemoto M, Hiraki Y (2004) Preoperative concurrent chemoradiotherapy with cisplatin and docetaxel in patients with locally advanced non-small-cell lung cancer. Br J Cancer 90: 979-984

Kawamura H, Terashima M, Ikeda K, Takiyama I, Sasaki N, Ishida K, Saito K (1997) Antitumour activities of Taxotere and Taxol against human esophageal cancer. Proc Amer Assoc Cancer Res 38: A1540

Kiura K, Ueoka H, Segawa Y, Tabata M, Kamei H, Takigawa N, Hiraki S, Watanabe Y, Bessho A, Eguchi K, Okimoto N, Harita S, Takemoto M, Hiraki Y, Harada M, Tanimoto M (2003) Phase I/II study of docetaxel and cisplatin with concurrent thoracic radiation therapy for locally advanced non-small-cell lung cancer. Br J Cancer 89: 795-802

Laack E, Andritzky B, Durk H, Burkholder I, Edler L, Schuch G, Boeters I, Gorn M, Lipp R, Horst H, Popp J, Hossfeld DK (2005) Docetaxel and cisplatin as first-line treatment for patients with metastatic esophageal cancer: a pilot study. Onkologie 28: 647-650

Lordick F, von Schilling C, Bernhard H, Hennig M, Bredenkamp R, Peschel C (2003) Phase II trial of irinotecan plus docetaxel in cisplatin-pretreated relapsed or refractory oesophageal cancer. $\mathrm{Br} J$ Cancer 89: 630 -633

Lorenzen S, Duyster J, Lersch C, von Delius S, Henning M, Bredenkamp R, Peschel C, Lordick F (2005) Capecitabine plus docetaxel every 3 weeks in first- and second-line metastatic oesophageal cancer: final results of a phase II trial. Br J Cancer 92: 2129-2133

Mason KA, Hunter NR, Milas M, Abbruzzese JL, Milas L (1997) Docetaxel enhances tumour radioresponse in vivo. Clin Cancer Res 3: 2431-2438

Mauer AM, Masters GA, Haraf DJ, Hoffman PC, Watson SM, Golomb HM, Vokes EE (1998) Phase I study of docetaxel with concomitant thoracic radiation therapy. J Clin Oncol 16: 159- 164
Mauer A, Haraf D, Ferguson M, Posner M, Sandler A, Kesler K, Hoffman P, Rudin C, Talabay K, Vokes E (2000) Docetaxel-based combined modality therapy for locally advanced carcinoma of the esophagus and gastric cardia. Proc Am Soc Clin Oncol 19: 2000 (abstract 954)

Meguid RA, Hooker CM, Taylor JT, Kleinberg LR, Cattaneo SM, Sussman MS, Yang SC, Heitmiller RF, Forastiere AA, Brock MV (2009) Recurrence after neoadjuvant chemoraditaion and surgery for esophageal cancer: Does the pattern of recurrence differ for patients with complete response and those with partial or no response? J Thorac Cardiovasc Surg 138: $1309-1317$

Mudad R, Ramsey M, Kovitz K, Curiel TJ, Hartz R, Nedzi LL, Weiner RS, Zakris EL (2003) Concomitant weekly docetaxel, cisplatin and radiation therapy in locally advanced non-small cell lung cancer: a dose finding study. Lung Cancer 39: 173 - 177

Pasini F, de Manzoni G, Pedrazzani C, Grandinetti A, Durante E, Gabbani M, Tomezzoli A, Griso C, Guglielemi A, Pelosi G, Maluta S, Cetto GL, Cordiano C (2005) High pathological response rate in locally advanced esophageal cancer after neoadjuvant combined modality therapy: dose finding of a weekly chemotherapy schedule with protracted venous infusion of 5 -fluorouracil and dose escalation of cisplatin, docetaxel and concurrent radiotherapy. Ann Oncol 16: 1133-1139

Ruhstaller T, Widmer L, Schuller JC, Roth A, Hess V, Mingrone W, von Moos R, Borner $\mathrm{M}$, Pestalozzi BC, BalmerMajno S, Köberle $\mathrm{E}$, Terraciano L, Schnider A, Bodis S, Popescu R (2009) Multicenter phase II trial of preoperative induction chemotherapy followed by chemoradiation with docetaxel and cisplatin for locally advanced esophageal carcinoma (SAKK 75/02). Ann Oncol 20: $1522-1528$

Wu H-G, Bang Y-J, Choi EK, Ahn YC, Kim YW, Lim T-H, Suh C, Park K, Park CI (2002) Phase I study of weekly docetaxel and cisplatin concurrent with thoracic radiotherapy in stage III non-small-cell lung cancer. Int J Rad Onc Biol Phys 52: 75-80 